TRANSACTIONS OF THE

AMERICAN MATHEMATICAL SOCIETY

Volume 353, Number 4 , Pages 1469-1487

S 0002-9947(00)02631-3

Article electronically published on September 13, 2000

\title{
THE AUTOMORPHISM GROUPS OF KUMMER SURFACES ASSOCIATED WITH THE PRODUCT OF TWO ELLIPTIC CURVES
}

\author{
JONGHAE KEUM AND SHIGEYUKI KONDŌ
}

\begin{abstract}
We calculate the automorphism groups of several Kummer surfaces associated with the product of two elliptic curves. We give their generators explicitly.
\end{abstract}

\section{INTRODUCTION}

The purpose of this paper is to calculate the automorphism groups of the following Kummer surfaces $X$ associated with the product of two elliptic curves:

Case I. $X=K m(E \times F)$, where $E$ and $F$ are non-isogenus generic elliptic curves.

Case II. $X=K m(E \times E)$, where $E$ is an elliptic curve without complex multiplications.

Case III. $X=K m\left(E_{\omega} \times E_{\omega}\right)$, where $\omega$ is a 3rd root of unity and $E_{\tau}$ is the elliptic curve with $\tau$ as its fundamental period.

Case IV. $X=K m\left(E_{\sqrt{-1}} \times E_{\sqrt{-1}}\right)$.

We shall give generators of the group of automorphisms explicitly (Theorems $5.3,5.4,5.6,5.7)$.

In the following we shall outline the proof of a theorem which is similar to that of Kondō [8] in which the second author calculated the group of automorphisms of a generic Kummer surface associated with a curve of genus two (for this case, also see Keum 5]). Recall that Kummer surfaces are $K 3$ surfaces. It follows from results of Piatetski-Shapiro and Shafarevich 13 that the group of automorphisms is isomorphic, up to finite groups, to the group of isometries of the Picard lattice $S_{X}$ of $X$ which preserve the Kähler cone $D(X)$ of $X$. In our cases, $A u t(X)$ is infinite (Keum [6]; also see Shioda and Inose [15] for Cases III, IV) and hence it is difficult to describe the Kähler cone explicitly.

On the other hand, we can embed $S_{X}$ into an even unimodular lattice $I I_{1,25}$ of signature $(1,25)$ such that the orthogonal complement $R$ of $S_{X}$ in $I I_{1,25}$ is a root lattice. Conway [3] determined a fundamental domain $D$ of the reflection group

Received by the editors May 30, 1999 and, in revised form, July 12, 1999.

2000 Mathematics Subject Classification. Primary 14J28, 14J50, 11 H56.

Key words and phrases. Automorphisms of Kummer surfaces, Picard lattice, Leech lattice.

The first author was supported by $\operatorname{KOSEF}(1999-2-102-002-3)$. The second author was supported in part by the Monbusho Grant-in Aid for Scientific Research (B) 10440005 and Houga 11874004. 
of $I I_{1,25}$ in terms of Leech roots. Following Borcherds [1, by restricting $D$ to the Picard lattice $S_{X}$, we can find a polyhedral cone $D^{\prime}$ in the Kähler cone $D(X)$ of $X$ bounded by finitely many faces. Since $R$ is a root lattice, we can easily calculate all faces of $D^{\prime}$ explicitly (Lemmas 3.2, 3.6). Next we shall carry out the most complicated part, i.e., for each face of $D^{\prime}$ we shall construct an automorphism of $X$ which works like a reflection with respect to this face $(\S 4)$. A standard argument shows that $A u t\left(D^{\prime}\right)$ and these automorphisms generate a subgroup of finite index in the orthogonal group $O\left(S_{X}\right)$.

Our method can be applied to other cases as long as the Picard lattice is the orthogonal complement of a root lattice in $I I_{1,25}$.

We remark that in Case I the group generated by all reflections of $S_{X}$ is of finite index in the orthogonal group of $S_{X}$, and in other cases it is not.

For basic definitions and results for lattices and Leech lattices, we refer the reader to the expository Sections 1 and 2 of the second author's previous paper 8$]$.

\section{Kummer SURFACES}

Let $E$ and $F$ be elliptic curves. Let $p_{i}, q_{i}(1 \leq i \leq 4)$ be 2 -torsion points of $E, F$ respectively. The quotient surface of $E \times F$ by the inversion $\left(-1_{E},-1_{F}\right)$ has sixteen ordinary nodes corresponding to sixteen 2-torsion points $\left(p_{i}, q_{j}\right)$ of $E \times F$. Let $X=K m(E \times F)$ be its minimal resolution, which is called the Kummer surface associated with $E \times F$.

It is well known that $X=K m(E \times F)$ is a $K 3$ surface, i.e. it is simply connected and its canonical line bundle is trivial. There is a canonical structure of lattice on $H^{2}(X, \mathbb{Z})$ with the cup product which is an even unimodular lattice of signature $(3,19)$, and hence isometric to $U^{\oplus 3} \oplus E_{8}^{\oplus 2}$, where $U$ is the even unimodular lattice of signature $(1,1)$ and $E_{8}$ the even unimodular lattice of signature $(0,8)$. Let $S_{X}$ be the Picard lattice of $X$ :

$$
S_{X}=\left\{x \in H^{2}(X, \mathbb{Z}):\left\langle x, \omega_{X}\right\rangle=0\right\},
$$

where $\omega_{X}$ is a nowhere vanishing holomorphic 2 -form on $X$. Let $T_{X}$ be the orthogonal complement of $S_{X}$, which is called the transcendental lattice of $X$. In our case

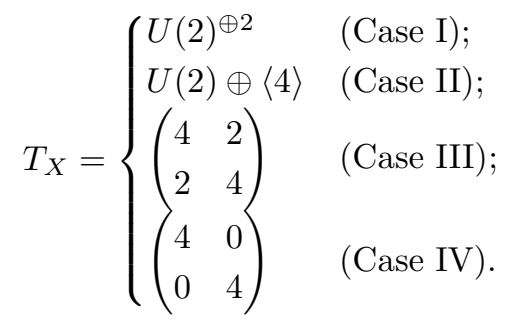

It follows from Nikulin [11, Theorem 3.1 that the restriction of $A u t(X)$ on $T_{X}$ is a direct sum of irreducible representations of a cyclic group of order $m$ defined over $\mathbb{Q}$ which has no nontrivial fixed vectors in $T_{X} \otimes \mathbb{C}$. If $\rho(X) \geq 18$, then this implies that $\varphi(m) \leq 4$, where $\varphi$ is the Euler function. In Case I, we call $X=K m(E \times F)$ generic if $m=2$.

Let $P\left(S_{X}\right)$ be the connected component of the cone $\left\{x \in S_{X} \otimes \mathbb{R}:\langle x, x\rangle>0\right\}$ which contains an ample divisor. Put

$$
D(X)=\left\{x \in P\left(S_{X}\right):\langle x, \delta\rangle \geq 0 \text { for all classes } \delta \text { of smooth rational curves }\right\},
$$


which is called the Kähler cone of $X$ and is a fundamental domain with respect to the natural action of $W\left(S_{X}\right)^{(2)}$ on $P\left(S_{X}\right)$. It follows from the Torelli theorem for $K 3$ surfaces due to Piatetski-Shapiro and Shafarevich [13] that $\operatorname{Aut}(X)$ is isomorphic to $O\left(S_{X}\right) /\left(W\left(S_{X}\right)^{(2)} \cdot\{ \pm 1\}\right)$ up to finite groups. Now we have

Lemma 1.1. In Case I, assume that $X$ is generic. Let

$$
G\left(S_{X}\right)=\left\{g \in O\left(S_{X}\right): g^{*} \mid A_{S_{X}}=1\right\} .
$$

Then Aut $(X)$ is an extension of $G\left(S_{X}\right) /\left(W\left(S_{X}\right)^{(2)} \cdot\{ \pm 1\}\right)$ by a cyclic group of order $m$, where $m=2,2,6$ or 4 for Case I, II, III or IV respectively.

\section{Picard lattice}

Let $E$ and $F$ be elliptic curves. Let $X=K m(E \times F)$ be the Kummer surface associated with $E \times F$. Obviously $X$ contains sixteen mutually disjoint smooth rational curves $\left\{G_{i j}\right\}$ obtained by resolution of singularities $\left(G_{i j}\right.$ corresponds to $\left.\left(p_{i}, q_{j}\right)\right)$. Let $E_{i}\left(\right.$ resp. $\left.F_{i}\right)$ be the image of $E \times\left\{q_{i}\right\}\left(\right.$ resp. $\left.\left\{p_{i}\right\} \times F\right)$. Then $\left\{E_{i}, F_{j}\right\}$ are mutually disjoint smooth rational curves on $X$. The incidence relation between these curves is

$$
G_{i j} \cdot E_{k}=\delta_{j, k}, \quad G_{i j} \cdot F_{k}=\delta_{i, k} .
$$

We assume that $E_{1}, E_{2}, E_{3}$ or $E_{4}$ (resp. $F_{1}, F_{2}, F_{3}$ or $F_{4}$ ) corresponds to $E \times\{0\}, E \times$ $\{1 / 2\}, E \times\left\{\tau^{\prime} / 2\right\}$ or $E \times\left\{\left(1+\tau^{\prime}\right) / 2\right\}$ respectively (resp. $\{0\} \times F,\{1 / 2\} \times F,\{\tau / 2\} \times F$ or $\{(1+\tau) / 2\} \times F$ ) where $\tau$ (resp. $\left.\tau^{\prime}\right)$ is the fundamental period of $E$ (resp. $F$ ).

In Cases II, III, IV, we denote by $D_{1}$ the nodal curve which is the image of the diagonal of $E \times E$, and by $D_{2}, D_{3}$ or $D_{4}$ the translation of $D_{1}$ by the 2-torsion point $(1 / 2,0),(\tau / 2,0)$ or $((\tau+1) / 2,0)$ respectively. We have two families of mutually disjoint nodal curves,

$$
\left\{G_{i j}\right\} \text { and }\left\{E_{i}, F_{j}, D_{k}\right\} .
$$

Each member of the first family (resp. the second one) meets 3 (resp. 4) members in the second one (resp. the first one):

$$
\begin{aligned}
& D_{1} \text { meets } G_{11}, G_{22}, G_{33}, G_{44} ; \\
& D_{2} \text { meets } G_{12}, G_{21}, G_{34}, G_{43} ; \\
& D_{3} \text { meets } G_{13}, G_{31}, G_{24}, G_{42} ; \\
& D_{4} \text { meets } G_{14}, G_{41}, G_{23}, G_{32}
\end{aligned}
$$

In Case III, further, we denote by $C_{i}$ the nodal curve which is the image of $\left\{p_{i}\right\} \times E_{\omega}$ under the map induced from the automorphism of $E_{\omega} \times E_{\omega}$ given by

$$
(x, y) \longrightarrow\left(\omega(-x+y),-\omega^{2} y\right) .
$$

We have two families consisting of sixteen mutually disjoint nodal curves,

$$
\left\{G_{i j}\right\} \text { and }\left\{E_{i}, F_{j}, D_{k}, C_{l}\right\},
$$

such that each member of one family meets exactly 4 members in the other family:

$$
\begin{aligned}
& C_{1} \text { meets } G_{11}, G_{23}, G_{34}, G_{42} ; \\
& C_{2} \text { meets } G_{14}, G_{22}, G_{31}, G_{43} ; \\
& C_{3} \text { meets } G_{12}, G_{24}, G_{33}, G_{41} ; \\
& C_{4} \text { meets } G_{13}, G_{21}, G_{32}, G_{44} .
\end{aligned}
$$


In Case IV, there exist, further, twelve nodal curves $\left\{L_{i}\right\}$ such that each member of $\left\{G_{i j}, E_{i}, F_{j}, D_{k}, L_{l}\right\}$ meets six members in this family. The configuration of such nodal curves was given in Kondō 7], Example IV (see Figure 4.3. Unfortunately there is a misprint in Figure 4.3. The incident relations of $E_{9}^{+}$and $E_{9}^{-}$(resp. $E_{10}^{+}$ and $\left.E_{10}^{-}\right)$with $E_{6}^{ \pm}, E_{8}^{ \pm}$should be changed). For example, $G_{11}, G_{12}, G_{13}, G_{14}, G_{21}$, $G_{22}, G_{23}, G_{24}, G_{31}, G_{32}, G_{33}, G_{34}, G_{41}, G_{42}, G_{43}, G_{44}$ correspond to $E_{11}^{+}, E_{12}^{+}$, $E_{10}^{+}, E_{9}^{+}, E_{11}^{-}, E_{12}^{-}, E_{10}^{-}, E_{9}^{-}, E_{14}^{+}, E_{13}^{-}, E_{15}^{-}, E_{16}^{+}, E_{13}^{+}, E_{14}^{-}, E_{16}^{-}, E_{15}^{+}$respectively; $E_{i}(1 \leq i \leq 4)$ correspond to $E_{1}^{+}, E_{3}^{+}, E_{2}^{-}, E_{4}^{-}$respectively; $F_{j}(1 \leq j \leq 4)$ correspond to $E_{17}^{+}, E_{17}^{-}, E_{20}^{+}, E_{20}^{-}$respectively; $D_{k}(1 \leq k \leq 4)$ correspond to $E_{5}^{-}$, $E_{7}^{+}, E_{8}^{+}, E_{6}^{-}$respectively; $L_{l}(1 \leq l \leq 12)$ correspond to $E_{2}^{+}, E_{4}^{+}, E_{19}^{-}, E_{19}^{+}, E_{6}^{+}$, $E_{8}^{-}, E_{5}^{+}, E_{7}^{-}, E_{18}^{-}, E_{18}^{+}, E_{3}^{-}, E_{1}^{-}$respectively. Here we assume that

$$
\begin{aligned}
& L_{1} \text { meets } G_{13}, G_{23}, G_{34}, G_{44}, E_{1}, E_{2} ; \\
& L_{2} \text { meets } G_{14}, G_{24}, G_{33}, G_{43}, E_{1}, E_{2} ; \\
& L_{3} \text { meets } G_{31}, G_{32}, G_{43}, G_{44}, F_{1}, F_{2} ; \\
& L_{4} \text { meets } G_{33}, G_{34}, G_{41}, G_{42}, F_{1}, F_{2} ; \\
& L_{5} \text { meets } G_{13}, G_{24}, G_{32}, G_{41}, D_{1}, D_{2} ; \\
& L_{6} \text { meets } G_{14}, G_{23}, G_{31}, G_{42}, D_{1}, D_{2} ; \\
& L_{7} \text { meets } G_{12}, G_{21}, G_{33}, G_{44}, D_{3}, D_{4} ; \\
& L_{8} \text { meets } G_{11}, G_{22}, G_{34}, G_{43}, D_{3}, D_{4} ; \\
& L_{9} \text { meets } G_{11}, G_{12}, G_{23}, G_{24}, F_{3}, F_{4} ; \\
& L_{10} \text { meets } G_{13}, G_{14}, G_{21}, G_{22}, F_{3}, F_{4} ; \\
& L_{11} \text { meets } G_{12}, G_{22}, G_{31}, G_{41}, E_{3}, E_{4} ; \\
& L_{12} \text { meets } G_{11}, G_{21}, G_{32}, G_{42}, E_{3}, E_{4} .
\end{aligned}
$$

Lemma 2.1. The above nodal curves generate the Picard lattice $S_{X}$.

Proof. First consider Case I. Let $\pi: X \longrightarrow \mathbb{P}^{1}$ be an elliptic fibration defined by the linear system $\left|2 E_{1}+G_{11}+G_{21}+G_{31}+G_{41}\right|$, which has four singular fibers of type $I_{0}^{*}$ (in Kodaira's sense) and four sections $F_{1}, F_{2}, F_{3}, F_{4}$. By Shioda [14], Corollaries $1.5,1.7$, the rank of the group of sections of $\pi$ is equal to 0 and its order is 4 . Since $S_{X}$ is generated by classes of irreducible components of fibres of $\pi$ and sections, we have the assersion for Case I. The remaining cases can be proved similarly by considering the following elliptic fibrations.

Case II. Consider the elliptic fibration defined by

$$
\left|G_{31}+G_{41}+G_{13}+G_{43}+2\left(E_{1}+G_{21}+F_{2}+G_{23}+E_{3}\right)\right|
$$

which has three singular fibers of type $I_{4}^{*}, I_{1}^{*}, I_{0}^{*}$ and two sections $F_{1}, F_{3}$;

Case III. Consider the elliptic fibration defined by

$$
\left|G_{11}+D_{1}+G_{44}+C_{4}+G_{13}+E_{3}+G_{23}+F_{2}+G_{24}+C_{3}+G_{41}+E_{1}\right|,
$$

which has four singular fibers of type $I_{12}, I_{4}, I_{3}, I_{3}$ and six sections $G_{12}, G_{31}, G_{32}, G_{43}$ plus two hidden ones.

Case IV. Consider the elliptic fibration defined by

$$
\left|E_{1}+E_{2}+L_{1}+L_{2}\right|
$$

which has six singular fibers of type $I_{4}$ and sixteen sections $\left\{G_{i j}\right\}$. 


\section{A relation between the Picard lattice and Leech roots}

Let $L$ be an even unimodular lattice of signature $(1,25)$, and $\Lambda$ the Leech lattice, that is, the even unimodular lattice of signature $(0,24)$ without a root. In the following we fix a decomposition $L=\Lambda \oplus U$, and take $w=(0,0,1)$ as a Weyl vector. We use the same notation as in Section 2 of [8]. Consider the following vectors in $\Lambda$ :

$$
\begin{gathered}
X=4 \nu_{\infty}+\nu_{\Omega}, \quad Y=4 \nu_{0}+\nu_{\Omega}, \quad Z=0, \\
P=4 \nu_{\infty}+4 \nu_{0}, \quad Q=\nu_{\Omega}-4 \nu_{2}, \\
R_{1}=2 \nu_{K_{1}}, \quad R_{2}=2 \nu_{K_{2}}, \\
R_{3}=\left(x_{\infty}, x_{0}, x_{1}, x_{2}, x_{j_{1}}, \ldots, x_{j_{4}}, x_{j_{5}}, \ldots, x_{j_{22}}\right)=(3,3,3,-1, \ldots,-1,1, \ldots, 1)
\end{gathered}
$$

where $K_{1}, K_{2}$ and $K_{3}=\left\{\infty, 0,1,2, j_{1}, \ldots, j_{4}\right\} \in \mathcal{C}(8), K_{1}, K_{2} \ni \infty, 0,1,2$.

Let

$$
\begin{gathered}
x=(X, 1,2), \quad y=(Y, 1,2), \quad z=(0,1,-1), \\
p=(P, 1,1), \quad q=(Q, 1,1), \\
r_{1}=\left(R_{1}, 1,1\right), \quad r_{2}=\left(R_{2}, 1,1\right), \quad r_{3}=\left(R_{3}, 1,2\right)
\end{gathered}
$$

be the corresponding Leech roots. We denote by $R$ the sublattice of $L$ generated by

Case I. $\left\{x, y, z, p, q, r_{1}, r_{2}, r_{3}\right\}$;

Case II. $\left\{x, y, z, p, q, r_{1}, r_{3}\right\}$;

Case III. $\left\{x, y, z, p, q, r_{3}\right\}$;

Case IV. $\left\{x, y, z, p, q, r_{1}\right\}$.

It is esay to see that $R$ is isometric to $D_{4} \oplus D_{4}, D_{4} \oplus A_{3}, D_{4} \oplus A_{2}$ or $A_{3} \oplus A_{3}$ for Case I, II, III or IV respectively.

Lemma 3.1. $R$ is a primitive sublattice of $L$.

Proof. Consider Case I. Note that $A_{R} \simeq(\mathbb{Z} / 2 \mathbb{Z})^{4}$ is generated by $(x-y) / 2$, $\left(x-r_{3}\right) / 2,\left(p-r_{1}\right) / 2,\left(p-r_{2}\right) / 2$. A direct calculation shows that $R^{*} \cap L=R$. This implies the assertion. The proofs of other cases are similar.

Let $S$ be the orthogonal complement of $R$ in $L$. Since $q_{R} \simeq q_{T_{X}}, S \simeq S_{X}$ (Nikulin [12], Theorem 1.14.2). Now put

$$
D^{\prime}=D \cap P(S)
$$

Then it is known that $D^{\prime}$ is non-empty, has only a finite number of faces, and is of finite volume. Moreover, $D^{\prime}$ contains the projection $w^{\prime}$ of the Weyl vector $w=(0,0,1) \in \Lambda \oplus U$ as an interior point (Borcherds [1], Lemmas 4.1, 4.2, 4.3). First we determine the Leech roots orthogonal to $R$.

Lemma 3.2. There are exactly twenty-four, twenty-eight, thirty-two or forty Leech roots which are orthogonal to $R$ for Case I, II, III or IV respectively.

Proof. We consider Case I only. The other cases can be proved similarly. By Conway and Sloane [4], Table 4.13, which is reformulated in [8] as Proposition 2.3 and Remark 2.4, we can see that such a Leech root $r$ corresponds to a vector $2 \nu_{K}$ 
in $\Lambda$, where $K \in \mathcal{C}(8), 0, \infty \in K, 2 \notin K,\left|K \cap K_{i}\right|=4, i=1,2$ and $K \cap K_{3}=\{\infty, 0\}$ or $\{\infty, 0,1, *\}$.

The number of these octads is counted as follows:

Case 1. $K \cap K_{3}=\{\infty, 0\}$. Take a point $A$ in $K_{1} \backslash K_{3}$ and a point $B$ in $K_{2} \backslash K_{3}$. Then the set $\{0, \infty, A, B\}$ determines a unique sextet, or, in other words, there exist exactly five octads containing $0, \infty, A, B$. Note that any two distinct octads meet in 0,2 or 4 points because the $\mathcal{C}$-set consists of $0,8,12,16$ or 24 elements. It follows easily from this fact that the above five octads satisfy the condition $\left|K \cap K_{i}\right|=4$ for $i=1,2$ and exactly two of them satisfy the condition $K \cap K_{3}=\{\infty, 0\}$. Let $K$ be a desired octad. Let $A^{\prime}$ be in $\Omega$ with $K \cap K_{1}=\left\{\infty, 0, A, A^{\prime}\right\}$. Then $K$ also appears when we consider the sextet defined by $\left\{\infty, 0, A^{\prime}, B\right\}$. Hence the number of desired octads is $\left(\left(\begin{array}{l}4 \\ 1\end{array}\right) \times\left(\begin{array}{l}4 \\ 1\end{array}\right) \times 2\right) /(2 \times 2)=8$.

Case 2. $K \cap K_{3}=\{\infty, 0,1, *\}$. Take a point $A$ in $K_{3} \backslash K_{1}$. Then $\{\infty, 0,1, A\}$ determines exactly five octads containing these four points. It is now easy to see that these five octads satisfy the condition $\left|K \cap K_{i}\right|=4$. Here we need to exclude $K_{3}$. Hence the desired number of octads is eqaul to $\left(\begin{array}{l}4 \\ 1\end{array}\right) \times(5-1)=16$.

Remark 3.3. In his paper 16, Todd gave the 759 octads of the Steiner system $S(5,8,24)$ (p. 219, Table I). The octads in the proof of Lemma 3.2 correspond to the following $\left\{E_{i}, F_{i}, G_{i j}\right\}$. Here we use the same symbol as nodal curves stated in $\S 2$ because these can be identified (see $\S 5$ ). We assume

$$
\begin{aligned}
& K_{1}=\{\infty, 0,1,2,3,5,14,17\}, \\
& K_{2}=\{\infty, 0,1,2,4,13,16,22\}, \\
& K_{3}=\{\infty, 0,1,2,6,7,19,21\} .
\end{aligned}
$$

Then

Case I.

$$
\begin{aligned}
G_{11} & =\{\infty, 0,1,3,4,11,19,20\}, \quad G_{21}=\{\infty, 0,1,3,6,8,10,13\}, \\
G_{12} & =\{\infty, 0,1,3,7,9,16,18\}, \quad G_{22}=\{\infty, 0,1,3,12,15,21,22\}, \\
G_{31} & =\{\infty, 0,1,4,5,7,8,15\}, \quad G_{14}=\{\infty, 0,1,4,6,9,12,17\}, \\
G_{34} & =\{\infty, 0,1,4,10,14,18,21\}, \quad G_{42}=\{\infty, 0,1,5,6,18,20,22\}, \\
G_{41} & =\{\infty, 0,1,5,9,11,13,21\}, \quad G_{32}=\{\infty, 0,1,5,10,12,16,19\}, \\
G_{33} & =\{\infty, 0,1,6,11,14,15,16\}, \quad G_{23}=\{\infty, 0,1,7,10,11,17,22\}, \\
G_{44} & =\{\infty, 0,1,7,12,13,14,20\}, \quad G_{43}=\{\infty, 0,1,8,9,14,19,22\}, \\
G_{13} & =\{\infty, 0,1,8,16,17,20,21\}, \quad G_{24}=\{\infty, 0,1,13,15,17,18,19\}, \\
E_{3} & =\{\infty, 0,3,4,5,12,13,18\}, \quad F_{4}=\{\infty, 0,3,4,10,15,16,17\}, \\
E_{4} & =\{\infty, 0,3,5,8,11,16,22\}, \quad F_{3}=\{\infty, 0,3,9,13,17,20,22\}, \\
F_{2} & =\{\infty, 0,4,5,9,14,16,20\}, \quad E_{2}=\{\infty, 0,4,8,11,13,14,17\}, \\
F_{1} & =\{\infty, 0,5,10,13,14,15,22\}, \quad E_{1}=\{\infty, 0,12,14,16,17,18,22\} ;
\end{aligned}
$$

Case II. $G_{i j}, E_{i}, F_{j}$ and

$$
\begin{aligned}
& D_{4}=\{\infty, 0,3,8,14,15,18,20\}, \quad D_{3}=\{\infty, 0,3,9,10,11,12,14\}, \\
& D_{1}=\{\infty, 0,5,8,9,10,17,18\}, \quad D_{2}=\{\infty, 0,5,11,12,15,17,20\} ;
\end{aligned}
$$

Case III. $G_{i j}, E_{i}, F_{j}, D_{k}$ and

$$
\begin{array}{ll}
C_{3}=\{\infty, 0,4,8,10,12,20,22\}, & C_{4}=\{\infty, 0,4,9,11,15,18,22\}, \\
C_{1}=\{\infty, 0,8,9,12,13,15,16\}, & C_{2}=\{\infty, 0,10,11,13,16,18,20\} ;
\end{array}
$$


Case IV. $G_{i j}, E_{i}, F_{j}, D_{k}$ and

$$
\begin{aligned}
L_{1} & =\{\infty, 0,3,5,6,9,15,19\}, \quad L_{2}=\{\infty, 0,3,5,7,10,20,21\}, \\
L_{3} & =\{\infty, 0,3,6,11,17,18,21\}, \quad L_{4}=\{\infty, 0,3,7,8,12,17,19\}, \\
L_{5} & =\{\infty, 0,3,4,6,7,14,22\}, \quad L_{6}=\{\infty, 0,3,13,14,16,19,21\}, \\
L_{7} & =\{\infty, 0,4,5,17,19,21,22\}, \quad L_{8}=\{\infty, 0,5,6,7,13,16,17\}, \\
L_{9} & =\{\infty, 0,5,6,8,12,14,21\}, \quad L_{10}=\{\infty, 0,5,7,11,14,18,19\}, \\
L_{11} & =\{\infty, 0,6,10,14,17,19,20\}, \quad L_{12}=\{\infty, 0,7,9,14,15,17,21\} .
\end{aligned}
$$

For simplicity we use the same symbols $E_{i}, F_{j}, G_{i j}, C_{k}, L_{l}$ for the corresponding Leech roots.

Lemma 3.4. Let $w^{\prime}$ be the projection of the Weyl vector $w=(0,0,1) \in \Lambda \oplus U$. Then

Case I. $w^{\prime}=\left(\sum 3\left(E_{i}+F_{i}\right)+\sum 2 G_{i j}\right) / 2,\left(w^{\prime}\right)^{2}=28$,

Case II. $\left.w^{\prime}=\left(\sum 6\left(E_{i}+F_{i}+D_{i}\right)+\sum 5 G_{i j}\right)\right) / 8,\left(w^{\prime}\right)^{2}=19$,

Case III. $w^{\prime}=\sum\left(E_{i}+F_{i}+D_{i}+C_{i}+G_{i j}\right) / 2,\left(w^{\prime}\right)^{2}=16$,

Case IV. $w^{\prime}=\sum\left(E_{i}+F_{i}+D_{i}+G_{i j}+L_{k}\right) / 4,\left(w^{\prime}\right)^{2}=10$.

Proof. We shall give a proof in Case I. The other cases are similar. It is easy to see that both sides of the equation in Lemma 3.4 have the self-intersection number 28 and meet all $E_{i}, F_{j}, G_{i j}$ with multiplicity one. Since $\left\{E_{i}, F_{j}, G_{i j}\right\}$ generate $S$ (Lemma 2.1), this implies the assertion.

We denote by $\operatorname{Aut}\left(D^{\prime}\right)$ the group of isometries of $S$ which preserves $D^{\prime}$. Let $\operatorname{Sym}(R)$ be the group of symmetries of the Dynkin diagram of $R$. Obviously the canonical map $\operatorname{Sym}(R) \longrightarrow O\left(q_{R}\right)$ is surjective. It follows from Nikulin [12, Proposition 1.6.1, that each isometry $\varphi \in \operatorname{Aut}\left(D^{\prime}\right)$ can be extended to an isometry of $L$ which preserves $D$. Hence $A u t\left(D^{\prime}\right)$ is isomorphic to the subgroup $G$ of $A u t(D)$ which preserves the Dynkin diagram of $R$. We denote by $G_{0}$ the subgroup of $G$ which acts trivially on $R$. We remark that if we identify the set of nodal curves given in $\S 2$ with the set of Leech roots in Lemma 3.2, then $G_{0}$ can be considered as a group of symplectic automorphisms of $X$, i.e. they act on $T_{X}$ trivially.

\section{Lemma 3.5.}

Case I. $G_{0} \simeq(\mathbb{Z} / 2 \mathbb{Z})^{4}, G / G_{0} \simeq O^{+}\left(4, \mathbb{F}_{2}\right) \simeq \operatorname{Sym}(R)$.

Case II. $G_{0} \simeq(\mathbb{Z} / 2 \mathbb{Z})^{4} \cdot \mathbb{Z} / 3 \mathbb{Z}, G / G_{0} \simeq S_{3} \times \mathbb{Z} / 2 \mathbb{Z} \simeq \operatorname{Sym}(R)$.

Case III. $G_{0} \simeq(\mathbb{Z} / 2 \mathbb{Z})^{4} \cdot A_{4}, G / G_{0} \simeq S_{3} \times \mathbb{Z} / 2 \mathbb{Z} \simeq \operatorname{Sym}(R)$.

Case IV. $G_{0} \simeq(\mathbb{Z} / 2 \mathbb{Z})^{4} \cdot A_{5}, G / G_{0} \simeq D_{8}$, dihedral of order $8, \simeq \operatorname{Sym}(R)$.

Proof. First consider Case I. Obviously the restriction of $G$ on $R$ is a subgroup of the symmetry group $\left(S_{3} \times S_{3}\right) \cdot \mathbb{Z} / 2 \mathbb{Z} \simeq O^{+}\left(4, \mathbb{F}_{2}\right)$ of the Dynkin diagram of $R$. $G_{0}$ is a subgroup of $M_{22}$ because $G_{0}$ fixes $X, Y, Z$ and $P$ (see Conway and Sloane [4], Chap.10, §3.5). Moreover, $G_{0}$ preserves three octads $K_{i}$ and fixes 1, 2. By the table of maximal subgroups of $M_{22}$ (Conway [2], Table 3), $G_{0}$ is isomorphic to a 2-elementary abelian group of order 16 . Thus $G$ is a subgroup of the group $(\mathbb{Z} / 2 \mathbb{Z})^{4} \cdot\left(\left(S_{3} \times S_{3}\right) \cdot \mathbb{Z} / 2 \mathbb{Z}\right)$. On the other hand, $O(S) \simeq O\left(S_{X}\right)$ contains a subgroup isomorphic to $(\mathbb{Z} / 2 \mathbb{Z})^{4} \cdot\left(\left(S_{3} \times S_{3}\right) \cdot \mathbb{Z} / 2 \mathbb{Z}\right)$ induced from the automorphism 
group $\operatorname{Aut}(\Gamma)$ of the graph $\Gamma$ whose vertices are $\left\{E_{i}, F_{j}, G_{i j}\right\}$, where $(\mathbb{Z} / 2 \mathbb{Z})^{4}$ is the group of translations by 2 -torsion points, $S_{3} \times S_{3}$ is generated by permutations on $\left\{E_{1}, E_{2}, E_{3}\right\}$ or $\left\{F_{1}, F_{2}, F_{3}\right\}$, and $\mathbb{Z} / 2 \mathbb{Z}$ is generated by an involution interchanging $E_{i}$ and $F_{i}$. By Lemma 3.4, they preserve $w^{\prime}$ and hence $D^{\prime}$. Thus $\operatorname{Aut}\left(D^{\prime}\right) \simeq G \simeq(\mathbb{Z} / 2 \mathbb{Z})^{4} \cdot\left(\left(S_{3} \times S_{3}\right) \cdot \mathbb{Z} / 2 \mathbb{Z}\right)$.

In Cases II, III, IV, the proof is similar. Here we only give generators of $G_{0}$ and $G / G_{0}$, and leave the details to the reader.

Case II. $G_{0}$ is generated by translations by 2 -torsions and an automorphism $\tau$ of order 3 induced from the automorphism of $E \times E$ given by

$$
(x, y) \longrightarrow(x-y, x) .
$$

$G / G_{0}$ is generated by an automorphism $\sigma$ which is induced from the automorphism of $E \times E$ given by

$$
(x, y) \longrightarrow(y, x)
$$

and the permutations on $\left\{E_{i}, F_{j}, D_{k}\right\}$ given by

$$
E_{1} \leftrightarrow E_{2}, \quad F_{1} \leftrightarrow F_{2}, \quad D_{3} \leftrightarrow D_{4},
$$

and

$$
E_{1} \leftrightarrow E_{3}, \quad F_{1} \leftrightarrow F_{3}, \quad D_{2} \leftrightarrow D_{4} .
$$

Case III. $G_{0}$ is generated by translations by 2-torsions and automorphisms induced from

$$
(x, y) \longrightarrow(y,-x+y)
$$

and

$$
(x, y) \longrightarrow\left(\omega(-x+y),-\omega^{2} y\right) .
$$

We remark that $A_{4}$ is isomorphic to the alternating group on the set of four families $\left\{E_{i}\right\},\left\{F_{j}\right\},\left\{D_{k}\right\},\left\{C_{l}\right\} . S_{3}$ is generated by an automorphism induced from that of $E_{\omega} \times E_{\omega}$ given by

$$
(x, y) \longrightarrow(\omega x, \omega y)
$$

and a transposition on the above set of four families, given by

$$
E_{2} \leftrightarrow E_{3}, \quad F_{3} \leftrightarrow F_{4} .
$$

$\mathbb{Z} / 2 \mathbb{Z}$ in $G / G_{0}$ is induced from an isometry $\sigma$ which interchanges $\left\{G_{i j}\right\}$ and $\left\{E_{i}\right.$, $\left.F_{j}, D_{k}, C_{l}\right\}$. Later we shall see that $\sigma$ is induced from an automorphism of $X$.

Case IV. From the configuration of forty nodal curves on $X$ one gets exactly five elliptic fibrations which has six singular fibers of type $I_{4}$ and sixteen sections, namely

$$
\begin{gathered}
\left|E_{1}+E_{2}+L_{1}+L_{2}\right|, \quad\left|G_{11}+G_{12}+F_{1}+L_{9}\right|, \quad\left|G_{11}+G_{21}+E_{1}+L_{12}\right|, \\
\left|G_{11}+G_{22}+D_{1}+L_{8}\right|, \quad\left|G_{33}+G_{44}+D_{1}+L_{7}\right| .
\end{gathered}
$$

The $G_{0}$ is generated by automorphisms induced from translations by sections of these elliptic fibrations. On the other hand, there exists a Leech root $r$ such that the Dynkin diagram of $\left\{x, y, z, p, q, r_{1}, r\right\}$ is isomorphic to $A_{7}$ or $D_{7}$ (see the following Lemma 3.6). By Borcherds [1, Lemma 9.6, Theorem 9.5, there is an isometry in $\operatorname{Aut}(D)$ whose restriction on $R$ is a symmetry of the Dynkin diagram of $R$. These two isometries generate $G / G_{0} \simeq D_{8}$. 
Next we shall classify the hyperplanes bounding $D^{\prime}$. It suffices to consider Leech roots $r$ such that $r$ and $R$ generate a root lattice $R^{\prime}$ with $\operatorname{rank}\left(R^{\prime}\right)=\operatorname{rank}(R)+1$, because otherwise the hyperplane orthogonal to $r$ does not meet $P(S)$. Obviously $R^{\prime}$ is isomorphic to

Case I. $D_{4} \oplus D_{4} \oplus A_{1}$ or $D_{4} \oplus D_{5}$;

Case II. $D_{4} \oplus A_{3} \oplus A_{1}, D_{4} \oplus D_{4}, D_{4} \oplus A_{4}, D_{5} \oplus A_{3}$ or $D_{8}$;

Case III. $D_{4} \oplus A_{2} \oplus A_{1}, D_{4} \oplus A_{3}, D_{5} \oplus A_{2}$ or $D_{7}$;

Case IV. $A_{3} \oplus A_{3} \oplus A_{1}, D_{4} \oplus A_{3}, A_{4} \oplus A_{3}, A_{7}$ or $D_{7}$.

We have already taken care of the first case $R^{\prime}=R \oplus A_{1}$ in Lemma 3.2.

Lemma 3.6. Let $r$ be a Leech root. We denote by $r^{\prime}$ the projection of $r$ into $S^{*}$. Then the number of Leech roots and $\left\langle r^{\prime}, r^{\prime}\right\rangle$ is as follows:

Case I-(1). $\left(R^{\prime}=D_{4} \oplus D_{5}\right): 24,\left\langle r^{\prime}, r^{\prime}\right\rangle=-1$.

Case II-(1). $\left(R^{\prime}=D_{4} \oplus D_{4}\right): 3,\left\langle r^{\prime}, r^{\prime}\right\rangle=-1$.

(2) $\left(R^{\prime}=D_{4} \oplus A_{4}\right): 8,\left\langle r^{\prime}, r^{\prime}\right\rangle=-5 / 4$.

(3) $\left(R^{\prime}=D_{5} \oplus A_{3}\right): 36,\left\langle r^{\prime}, r^{\prime}\right\rangle=-1$.

(4) $\left(R^{\prime}=D_{8}\right): 72,\left\langle r^{\prime}, r^{\prime}\right\rangle=-1 / 4$.

Case III-(1). $\left(R^{\prime}=D_{4} \oplus A_{3}\right): 8,\left\langle r^{\prime}, r^{\prime}\right\rangle=-4 / 3$.

(2) $\left(R^{\prime}=D_{5} \oplus A_{2}\right): 72,\left\langle r^{\prime}, r^{\prime}\right\rangle=-1$.

(3) $\left(R^{\prime}=D_{7}\right): 96,\left\langle r^{\prime}, r^{\prime}\right\rangle=-1 / 3$.

Case IV-(1). $\left(R^{\prime}=D_{4} \oplus A_{3}\right): 40,\left\langle r^{\prime}, r^{\prime}\right\rangle=-1$.

(2) $\left(R^{\prime}=A_{4} \oplus A_{3}\right): 64,\left\langle r^{\prime}, r^{\prime}\right\rangle=-5 / 4$.

(3) $\left(R^{\prime}=A_{7}\right): 160,\left\langle r^{\prime}, r^{\prime}\right\rangle=-1 / 2$.

(4) $\left(R^{\prime}=D_{7}\right): 320,\left\langle r^{\prime}, r^{\prime}\right\rangle=-1 / 4$.

Proof. First we shall give a proof in Case I. First assume that $\langle r, p\rangle=1$. Then by 8], Proposition 2.3 and Remark 2.4, the root $r$ corresponds to a vector

$$
Q^{\prime}=\nu_{\Omega}-4 \nu_{k}
$$

in $\Lambda$, where $k \notin K_{1}, K_{2}, k \in K_{3}$. Hence the number of such Leech roots is equal to 4 . Next, for a Leech root $r$ with $\langle r, p\rangle=1$, write $r=r^{\prime}+r^{\prime \prime}$, where $r^{\prime} \in S^{*}, r^{\prime \prime} \in R^{*}$. Then $r^{\prime \prime}=p^{*}=\left(-2 p-2 q-r_{1}-r_{2}\right) / 2$. Hence $\left\langle r^{\prime}, r^{\prime}\right\rangle=-1$. Finally, note that $A u t\left(D^{\prime}\right)$ acts on the set of vectors $\left\{x, y, p, r_{1}, r_{2}, r_{3}\right\}$ transitively (Lemma 3.5). Therefore the total number of Leech roots as above is equal to $4 \times 6=24$.

In the remaining cases the proof is similar. We shall give one such Leech root and leave the details to the reader. Here we use the same identification as in Remark 3.3 .

Case I. $r$ corresponds to $\nu_{\Omega}-4 \nu_{19}$. $r$ meets exactly four Leech roots $G_{i j}$, ij $=$ $11,24,32,43$. The projection $r^{\prime}$ is equal to

$$
\left(\sum\left(E_{i}+F_{i}\right)+\sum G_{i j}-2\left(G_{11}+G_{24}+G_{32}+G_{43}\right)\right) / 4 .
$$

Case II-(1). $r$ corresponds to $2 \nu_{K}, K=\{\infty, 0,1,2,4,13,16,22\} . r$ meets exactly four Leech roots $D_{i}, 1 \leq i \leq 4$. The projection $r^{\prime}$ is equal to

$$
\left(2 \sum\left(E_{i}+F_{i}+D_{i}\right)+\sum G_{i j}-4 \sum D_{i}\right) / 8 \text {. }
$$


II-(2): The same Leech root as in Case I. $r$ meets exactly four Leech roots $G_{i j}$, $i j=11,24,32,43$. The projection $r^{\prime}$ is equal to

$$
\left(2 \sum\left(E_{i}+F_{i}+D_{i}\right)+3 \sum G_{i j}-8\left(G_{11}+G_{24}+G_{32}+G_{43}\right)\right) / 16 .
$$

II-(3): $r$ corresponds to

$$
\left(x_{\infty}, x_{2}, x_{4}, x_{6}, x_{7}, x_{8}, x_{12}, x_{13}, \ldots\right)=(3,-1,-1,-1,-1,-1,-1,-1,1, \ldots, 1),
$$

where $K=\{\infty, 2,4,6,7,8,12,13\}$ is an octad. $r$ meets exactly six Leech roots $G_{i j}, E_{k}, i j=14,21,31,44, k=2,3$. The projection of $r^{\prime}$ is equal to

$$
\left(\sum\left(F_{i}+D_{i}\right)+\sum G_{i j}-2\left(G_{14}+G_{21}+G_{31}+G_{44}\right)\right) / 4
$$

II-(4): $r$ corresponds to $2 \nu_{K}, K=\{\infty, 1,3,4,5,9,10,22\} . r$ meets exactly eight Leech roots $G_{i j}, E_{k}, D_{l}, i j=13,24,33,44, k=1,2, l=2$, 4 . The projection $r^{\prime}$ is equal to

$$
\begin{aligned}
& \left(6 \sum\left(E_{i}+F_{i}+D_{i}\right)+5 \sum G_{i j}-8\left(E_{1}+E_{2}+D_{2}+D_{4}\right)\right. \\
& \left.\quad-4\left(G_{12}+G_{13}+G_{21}+G_{24}+G_{32}+G_{33}+G_{41}+G_{44}\right)\right) / 16 .
\end{aligned}
$$

Case III-(1). The same Leech root as in Case I. $r$ meets exactly four Leech roots $G_{i j}, i j=11,24,32,43$. The projection $r^{\prime}$ is equal to

$$
\left(\sum\left(E_{i}+F_{i}+D_{i}+C_{i}\right)+2 \sum G_{i j}-6\left(G_{11}+G_{24}+G_{32}+G_{43}\right)\right) / 12 .
$$

III-(2): The same Leech root as in Case II-(3). $r$ meets exactly eight Leech roots $G_{i j}, E_{k}, C_{l}, i j=14,21,31,44, k=2,3, l=1,3$. The projection $r^{\prime}$ is equal to

$$
\left(\sum\left(F_{i}+D_{i}\right)+\sum G_{i j}-2\left(G_{14}+G_{21}+G_{31}+G_{44}\right)\right) / 4 \text {. }
$$

III-(3): The same Leech root as in Case II-(4). $r$ meets exactly ten Leech roots $G_{i j}, E_{k}, D_{l}, C_{m}, i j=13,24,33,44, k=1,2, l=2,4, m=1,2$. The projection $r^{\prime}$ is equal to

$$
\left(2 \sum\left(E_{i}+F_{i}+D_{i}+C_{i}\right)+\sum G_{i j}-3\left(E_{1}+E_{2}+D_{2}+D_{4}+C_{1}+C_{2}\right)\right) / 6 .
$$

Case IV-(1). The same Leech root as in Case II-(1). $r$ meets exactly twelve Leech roots $D_{i}, 1 \leq i \leq 4, L_{j}, j=1,2,3,4,9,10,11,12$. The projection $r^{\prime}$ is equal to

$$
\left(2 \sum\left(E_{i}+F_{i}+D_{i}\right)+\sum G_{i j}-4 \sum D_{i}\right) / 8 \text {. }
$$

IV-(2): The same Leech root as in Case I. $r$ meets exactly ten Leech roots $G_{i j}, L_{k}, i j=11,24,32,43, k=1,4,6,7,10,11$. The projection $r^{\prime}$ is equal to

$\left(2 \sum\left(E_{i}+F_{i}+D_{i}\right)+4 \sum L_{j}+\sum G_{i j}-8\left(L_{1}+L_{4}+L_{6}+L_{7}+L_{10}+L_{11}\right)\right) / 16$.

IV-(3): The same Leech root as in Case II-(4). $r$ meets exactly sixteen Leech roots $G_{i j}, E_{k}, D_{l}, L_{m}, i j=13,24,33,44, k=1,2, l=2,4, m=3,4,6,8,9,10,11$, 12. The projection $r^{\prime}$ is equal to

$$
\begin{aligned}
& \left(2 \sum G_{i j}+\sum\left(E_{i}+F_{i}+D_{i}\right)-\sum L_{j}\right. \\
& \quad-2\left(G_{11}+G_{22}+G_{31}+G_{42}+G_{13}+G_{24}+G_{33}+G_{44}\right) \\
& \left.\quad+4\left(L_{1}+L_{2}+L_{5}+L_{7}\right)\right) / 8
\end{aligned}
$$

IV-(4): $r$ corresponds to

$$
\left(x_{\infty}, x_{1}, x_{4}, x_{6}, x_{7}, x_{10}, x_{16}, x_{20}, \ldots\right)=(3,-1,-1,-1,-1,-1,-1,-1,1, \ldots, 1),
$$


where $K=\{\infty, 1,4,6,7,10,16,20\}$ is an octad. The Leech root $r$ meets exactly eighteen Leech roots $G_{i j}, F_{k}, L_{l}, i j=11,12,13,14,21,23,31,32,33,34,42,44$, $k=2,4, l=2,5,8,11$. The projection $r^{\prime}$ is equal to

$$
\begin{gathered}
\left(5 \sum G_{i j}+20 \sum F_{i}+10 \sum L_{i}-16\left(G_{22}+G_{24}+G_{41}+G_{43}\right)\right. \\
\left.-24\left(F_{2}+F_{4}+L_{2}+L_{5}+L_{8}+L_{11}\right)\right) / 16 .
\end{gathered}
$$

\section{EXAmples of AUtomorphisms of $X$}

In this section, we shall give examples of automorphisms of $X$ corresponding to the Leech roots listed in Lemma 3.6 (see also the proof of Lemma 3.6).

4.1. Case I. Put

$$
H=\left(\sum_{i}\left(E_{i}+F_{i}\right)+\sum_{i, j} G_{i j}\right) / 2 .
$$

Then $H^{2}=4, H \cdot E_{i}=H \cdot F_{j}=1$ and $H \cdot G_{i j}=0$ for any $i, j$. Recall that $2 E_{i}+\sum_{j} G_{j i}$ (resp. $2 F_{j}+\sum_{i} G_{j i}$ ) is a singular fiber of an elliptic fibration (see the proof of Lemma 2.1). From this fact we can easily see that $H \in S_{X}$. Put

$$
r^{\prime}=\left(H-G_{i_{1} j_{1}}-G_{i_{2} j_{2}}-G_{i_{3} j_{3}}-G_{i_{4} j_{4}}\right) / 2,
$$

where $\left\{i_{1}, i_{2}, i_{3}, i_{4}\right\}=\left\{j_{1}, j_{2}, j_{3}, j_{4}\right\}=\{1,2,3,4\}$. Obviously $\left\langle r^{\prime}, r^{\prime}\right\rangle=-1$, and the number of vectors $r^{\prime}$ of this form is 24 . These $r^{\prime}$ correspond to the Leech roots in Lemma 3.6. For each $r^{\prime}$ we shall construct an involution $f_{r^{\prime}}$ which sends $r^{\prime}$ to $-r^{\prime}$. For simplicity we may assume that $r^{\prime}=\left(H-G_{12}-G_{21}-G_{34}-G_{43}\right) / 2$.

First consider the elliptic pencil $\pi: X \longrightarrow \mathbb{P}^{1}$ defined by the linear system

$$
\left|G_{13}+G_{14}+G_{31}+G_{41}+2\left(G_{11}+E_{1}+F_{1}\right)\right| \text {. }
$$

Then $\pi$ has two singular fibers of type $I_{2}^{*}$ in Kodaira's sense, and $G_{33}, G_{34}, G_{43}$, $G_{44}$ are components of other singular fibers. Since $E_{3}, E_{4}, F_{3}, F_{4}$ are sections of $\pi$, other singular fibers are all of type $I_{2}$ or $I I I$. It follows from Shioda 14, Corollaries $1.5,1.7$, that the Mordell-Weil group of $\pi$ is isomorphic to $(\mathbb{Z} / 2 \mathbb{Z})^{2}$. Hence the translation sending $E_{4}$ to $F_{4}$ acts on $X$ as an involution $f_{r^{\prime}}$. The action of $f_{r^{\prime}}$ on twenty-four nodal curves is as follows:

$$
\begin{aligned}
& f_{r^{\prime}}\left(E_{i}\right)=F_{i}, \\
& f_{r^{\prime}}\left(G_{i j}\right)= \begin{cases}G_{i i}, & \text { if } i=j ; \\
G_{j i}, & \text { if }(i, j) \notin\{(1,2),(2,1),(3,4),(4,3)\} ; \\
G_{j i}+2 r^{\prime}, & \text { if }(i, j) \in\{(1,2),(2,1),(3,4),(4,3)\} .\end{cases}
\end{aligned}
$$

It is easy to see that $f_{r^{\prime}}(H)=H+4 r^{\prime}$ and $f_{r^{\prime}}\left(r^{\prime}\right)=-r^{\prime}$.

Remark 4.1. Take an involution $t$ induced from the translation of $E \times F$ by 2torsion with $t\left(E_{1}\right)=E_{2}, t\left(F_{1}\right)=F_{2}$. Let $\iota$ be the involution of $X$ induced from an automorphism of $E \times F$ given by $(x, y) \longrightarrow(x,-y)$. We shall see that $t \circ f_{r^{\prime}}$ comes from a Cremona transformation of $\mathbb{P}^{2}$. Recall that the linear system $|H|$ gives a double cover $\pi: X \longrightarrow Q=\mathbb{P}^{1} \times \mathbb{P}^{1}$ which is the composite of the quotient map by $\iota$ and contractions of sixteen nodal curves $G_{i j}$. Put $w_{i j}=\pi\left(G_{i j}\right)$. We use the same symbols $E_{i}, F_{j}$ for the images of $E_{i}, F_{j}$ in $Q$. Then $w_{i j}$ is the intersection point of $E_{j}$ and $F_{i}$. First blow up at $w_{21}$ and then contract the proper transform of 
$E_{1}$ and $F_{2}$. Then we have seven lines $\left\{\bar{E}_{2}, \bar{E}_{3}, \bar{E}_{4}, \bar{F}_{1}, \bar{F}_{3}, \bar{F}_{4}, L\right\}$ in $\mathbb{P}^{2}$, where $\bar{E}_{i}, \bar{F}_{j}$ are the image of the proper transforms of $E_{i}, F_{j}$ respectively and $L$ is the image of the exceptional divisor appearing at the first blow-up. Three lines $\bar{E}_{2}, \bar{E}_{3}, \bar{E}_{4}$ meet at one point (= the image of $F_{2}$ ), $\bar{F}_{1}, \bar{F}_{3}, \bar{F}_{4}$ meet at one point (= the image of $E_{1}$ ), and $L$ passes through these two points. Now consider the standard Cremona transformation of $\mathbb{P}^{2}$ with base $w_{12}, w_{34}, w_{43}$ which induces a birational involution $c_{r^{\prime}}$ of $Q$. It is easy to see that $c_{r^{\prime}}$ preserves the divisor $\sum_{i}\left(E_{i}+F_{i}\right)$, and hence it can be lifted to an involution $\tau_{r^{\prime}}$ of $X$. We can easily see that the actions of $\tau_{r^{\prime}}$ and $t \circ f_{r^{\prime}}$ on $S_{X}$ coincide, and hence $\tau_{r^{\prime}} \equiv t \circ f_{r^{\prime}} \bmod \iota$ by the Torelli theorem for $K 3$ surfaces (Piatetski-Shapiro and Shafarevich [13]).

Remark 4.2. The reflection associated with the $(-1)$-vector $r^{\prime}$ is not represented by an automorphism of $X$ because it does not act on $A_{S_{X}}$ as \pm 1 .

Remark 4.3. The description of $X$ as a double plane branched along the union of two sets of three lines meeting at one point shows that $X$ is a degeneration of a Jacobian Kummer surface which is a Kummer surface associated with a curve of genus two. There are sixteen projections of a Jacobian Kummer surface onto $\mathbb{P}^{2}$, resulting in sixteen involutions called projections [5]. These sixteen involutions on a Jacobian Kummer surface become the same involution $\iota$ on $X$.

4.2. Case II-(1). We assume that

$$
r^{\prime}=\left(2 \sum\left(E_{i}+F_{i}+D_{i}\right)+\sum G_{i j}-4 \sum D_{i}\right) / 8
$$

Put

$$
H=\left(\sum 2\left(E_{i}+F_{i}+D_{i}\right)+\sum G_{i j}\right) / 4 .
$$

It is easy to see that $H^{2}=4, H \cdot G_{i j}=1, H \cdot E_{i}=H \cdot F_{i}=H \cdot D_{i}=0$ and $H$ is in $S_{X}$. Let $f_{r^{\prime}}$ be an involution of $X$ induced from the involution $\left(1_{E},-1_{E}\right)$ of $E \times E$. Then $f_{r^{\prime}}$ preserves each curve in $\left\{G_{i j}, E_{i}, F_{j}\right\}$ and

$$
f_{r^{\prime}}\left(D_{i}\right)=H-\sum_{j \neq i} D_{j} .
$$

Thus $f_{r^{\prime}}$ acts on $S_{X}$ as a reflection with respect to the $(-4)$-vector $2 r^{\prime}=H-\sum_{i} D_{i}$.

Case II-(2). We assume that

$$
r^{\prime}=\left(2 \sum\left(E_{i}+F_{i}+D_{i}\right)+3 \sum G_{i j}-8\left(G_{11}+G_{24}+G_{32}+G_{43}\right)\right) / 16 .
$$

We shall find an automorphism $f_{r^{\prime}}$ of $X$ such that $f_{r^{\prime}}\left(r^{\prime}\right)=-s^{\prime}$ where

$$
s^{\prime}=\left(2 \sum\left(E_{i}+F_{i}+D_{i}\right)+3 \sum G_{i j}-8\left(G_{11}+G_{23}+G_{34}+G_{42}\right)\right) / 16
$$

is the projection of a Leech root $s$ of the same type.

Consider the elliptic pencil $\pi$ on $X$ defined by the linear system

$$
\begin{aligned}
\left|G_{21}+F_{2}+G_{22}+E_{2}+G_{12}+D_{2}\right| & =\left|G_{31}+F_{3}+G_{33}+E_{3}+G_{13}+D_{3}\right| \\
& =\left|G_{41}+F_{4}+G_{44}+E_{4}+G_{14}+D_{4}\right| .
\end{aligned}
$$

The elliptic pencil $\pi$ has three singular fibers of type $I_{6}$ and a singular fiber of type $I_{2}$ containg $G_{11}$, and has nine sections $E_{1}, F_{1}, D_{1}, G_{23}, G_{24}, G_{32}, G_{34}, G_{42}, G_{43}$. We remark that its Mordell-Weil group has rank 1 . We consider $E_{1}$ as 0 -section of $\pi$. Then the translation by $G_{43}$ induces an automorphism $f_{r^{\prime}}$ of $X$ of infinite order. Since $E_{1} \cdot G_{43}=0, G_{43} \cdot f_{r^{\prime}}\left(G_{43}\right)=0$. Also $E_{1} \cdot f_{r^{\prime}}\left(G_{43}\right)=0$ because $f_{r^{\prime}}$ 
has infinite order, and hence $f_{r^{\prime}}^{2}$ acts on smooth locus of each fiber freely. This determines $f_{r^{\prime}}\left(G_{43}\right)$, i.e.

$$
\begin{aligned}
f_{r^{\prime}}\left(G_{43}\right)= & G_{11}-G_{23}+\left(G_{13}+G_{14}+G_{21}+G_{22}+G_{33}+G_{41}-G_{34}-G_{42}\right) / 2 \\
& +E_{1}+F_{1}+D_{1} .
\end{aligned}
$$

Similary we have

$$
\begin{aligned}
f_{r^{\prime}}\left(F_{1}\right)= & G_{32}, f_{r^{\prime}}\left(D_{1}\right)=G_{24}, f_{r^{\prime}}\left(G_{42}\right)=D_{1}, f_{r^{\prime}}\left(G_{23}\right)=E_{1}, f_{r^{\prime}}\left(G_{34}\right)=F_{1}, \\
f_{r^{\prime}}\left(G_{32}\right)= & G_{11}-G_{34}+\left(G_{12}+G_{14}+G_{21}+G_{31}+G_{33}+G_{44}-G_{23}-G_{42}\right) / 2 \\
& +E_{1}+F_{1}+D_{1}, \\
f_{r^{\prime}}\left(G_{24}\right)= & G_{11}-G_{42}+\left(G_{12}+G_{13}+G_{22}+G_{31}+G_{41}+G_{44}-G_{23}-G_{34}\right) / 2 \\
& +E_{1}+F_{1}+D_{1} .
\end{aligned}
$$

A direct calculation shows that $f_{r^{\prime}}\left(r^{\prime}\right)=-s^{\prime}$. We remark that $f_{s^{\prime}}$ is given by the translation by $G_{23}$, and $f_{r^{\prime}}^{-1}=f_{s^{\prime}}$.

Case II-(3). We assume that

$$
r^{\prime}=\left(\sum\left(F_{i}+D_{i}\right)+\sum G_{i j}-2\left(G_{14}+G_{21}+G_{31}+G_{44}\right)\right) / 4
$$

By interchanging $E_{i}$ and $D_{i}$, we have the same situation as in Case I. Let $\pi$ be the elliptic pencil on $X$ defined by the linear system

$$
\begin{aligned}
\mid G_{12} & +G_{13}+G_{22}+G_{33}+2\left(F_{1}+D_{1}+G_{11}\right) \mid \\
& =\left|G_{23}+G_{32}+G_{42}+G_{43}+2\left(F_{4}+D_{4}+G_{41}\right)\right|=\left|G_{21}+G_{21}^{\prime}\right| \\
& =\left|G_{31}+G_{31}^{\prime}\right|=\left|G_{24}+G_{34}+E_{4}+E_{4}^{\prime}\right|,
\end{aligned}
$$

where $G_{21}^{\prime}, G_{31}^{\prime}, E_{4}^{\prime}$ are nodal curves. $\pi$ has two singular fibers of type $I_{2}^{*}$, two of type $I_{2}$ and one of type $I_{4}$, and its Mordell-Weil group is $(\mathbb{Z} / 2 \mathbb{Z})^{2}$. Let $f_{r^{\prime}}$ be an involution induced from the translation by sending $F_{2}$ to $F_{3}$. We can easily see that

$$
G_{21}^{\prime}=G_{31}+2 r^{\prime}, \quad G_{31}^{\prime}=G_{21}+2 r^{\prime}, \quad G_{14}^{\prime}=G_{14}+2 r^{\prime}, \quad G_{44}^{\prime}=G_{44}+2 r^{\prime} .
$$

Thus we have $f_{r^{\prime}}\left(r^{\prime}\right)=-r^{\prime}$.

Case II-(4). We assume that

$$
\begin{aligned}
r^{\prime}= & \left(6 \sum\left(E_{i}+F_{i}+D_{i}\right)+5 \sum G_{i j}-8\left(E_{1}+E_{2}+D_{2}+D_{4}\right)\right. \\
& \left.-4\left(G_{12}+G_{13}+G_{21}+G_{24}+G_{32}+G_{33}+G_{41}+G_{44}\right)\right) / 16 .
\end{aligned}
$$

Consider the following twenty nodal curves which are orthogonal to $r^{\prime}: G_{i j}, E_{k}$, $F_{l}, D_{m}, i j=11,12,14,21,22,23,31,32,34,41,42,43, k=3,4, l=1,2,3,4$, $m=1,3$. The dual graph of these curves except $G_{12}, G_{21}, G_{32}, G_{41}$ is the same as that of singular fiber of an elliptic pencil $\pi$ of type $I_{16}$, and $G_{12}, G_{21}, G_{32}, G_{41}$ are sections of $\pi$. This diagram appears in the classification of cohomologically trivial automorphisms of Enriques surfaces (Mukai and Namikawa [10], 4.2; also see Kondō [7], 1.7). The linear system

$$
\begin{aligned}
|H|=\mid & G_{21}+G_{23}+G_{31}+G_{32}+2\left(G_{12}+G_{22}+G_{34}+E_{4}+F_{2}+F_{3}+D_{1}\right) \\
& +3\left(G_{11}+G_{14}\right)+4 F_{1} \mid
\end{aligned}
$$

gives a morphism of degree 2 from $X$ to a smooth quadric $\mathbb{P}^{1} \times \mathbb{P}^{1}\left(E_{3}, E_{4}, D_{1}, D_{3}\right.$ are rulings). Denote by $f_{r^{\prime}}$ its covering transformation. Since $X /\left\langle f_{r^{\prime}}\right\rangle$ is rational, 
$f_{r^{\prime}}$ acts on $H^{0}\left(X, \Omega_{X}^{2}\right)$ as -1 . This implies that the fixed locus of $f_{r^{\prime}}$ is a disjoint union of smooth curves. Obviously $f_{r^{\prime}}$ fixes $F_{1}, F_{2}, F_{3}, F_{4}, E_{3}, E_{4}, D_{1}, D_{3}$, and acts on each $G_{i j}, i j=11,12,14,21,22,23,31,32,34,41,42,43$, as an involution. Thus $f_{r^{\prime}}$ acts trivially on the sublattice of rank 18 generated by these nodal curves. Since $f_{r^{\prime}}$ acts on a section as involution, there is a unique other fiber $F$ of $\pi$ invariant under $f_{r^{\prime}}$. Therefore $f_{r^{\prime}}$ fixes a component $C$ of $F$ which meets $G_{i j}, i j=12,21,32$ or 41. We shall see that $C$ is a smooth elliptic curve. Assume that $F$ is reducible. Then it is of type $I_{2}$ or $I I I$ because the Picard number of $X$ is 19 . Thus $C \cdot G_{i j}=1$ for all $i j=12,21,32,41$, and hence the image of $C$ in $\mathbb{P}^{1} \times \mathbb{P}^{1}$ is a smooth divisor of bidegree $(2,2)$. This is a contradiction. Hence $C=F$ is smooth. Thus we have the same configuration of fixed curves as in Mukai and Namikawa 10, (4.2). Since $f_{r^{\prime}}$ acts on the orthogonal complement of this sublattice as -1 (Mukai and Namikawa [10], Proposition 2.5), $f_{r^{\prime}}$ acts on $S_{X}$ as a reflection with respect to $r^{\prime}$.

4.3. Case III-(1). We assume that

$$
r^{\prime}=\left(\sum\left(E_{i}+F_{i}+D_{i}+C_{i}\right)+2 \sum G_{i j}-6\left(G_{11}+G_{24}+G_{32}+G_{43}\right)\right) / 12 .
$$

This case is similar to Case II-(2). We shall define an automorphism $f_{r^{\prime}}$ such that $f_{r^{\prime}}\left(r^{\prime}\right)=-s^{\prime}$, where

$$
s^{\prime}=\left(\sum 2\left(E_{i}+F_{i}+D_{i}+C_{i}\right)+\sum G_{i j}-6\left(C_{1}+C_{2}+C_{3}+C_{4}\right)\right) / 12
$$

is the projection of a Leech root $s$ of the same type. Consider the elliptic pencil defined by the same linear system as in Case II-(2). This elliptic pencil has three singular fibers of type $I_{6}$ and one singular fiber of type $I_{3}$ containing $G_{11}, C_{1}$, and twelve sections. The translation by sending $E_{1}$ to $G_{43}$ induces an automorphism $f_{r^{\prime}}$ :

$$
\begin{aligned}
f_{r^{\prime}}\left(C_{2}\right)= & G_{23}, \quad f_{r^{\prime}}\left(C_{3}\right)=G_{42}, \quad f_{r^{\prime}}\left(C_{4}\right)=G_{34}, \\
f_{r^{\prime}}\left(G_{43}\right)= & G_{11}-G_{23}+\left(G_{13}+G_{14}+G_{21}+G_{22}+G_{33}+G_{41}-G_{34}-G_{42}\right) / 2 \\
& +E_{1}+F_{1}+D_{1}-C_{1}, \\
f_{r^{\prime}}\left(G_{32}\right)= & G_{11}-G_{34}+\left(G_{12}+G_{14}+G_{21}+G_{31}+G_{33}+G_{44}-G_{23}-G_{42}\right) / 2 \\
& +E_{1}+F_{1}+D_{1}-C_{1}, \\
f_{r^{\prime}}\left(G_{24}\right)= & G_{11}-G_{42}+\left(G_{12}+G_{13}+G_{22}+G_{31}+G_{41}+G_{44}-G_{23}-G_{34}\right) / 2 \\
& +E_{1}+F_{1}+D_{1}-C_{1} .
\end{aligned}
$$

A direct calculation shows that $f_{r^{\prime}}\left(r^{\prime}\right)=-s^{\prime}$.

Case III-(2). We assume that

$$
r^{\prime}=\left(\sum\left(F_{i}+D_{i}\right)+\sum G_{i j}-2\left(G_{14}+G_{21}+G_{31}+G_{44}\right)\right) / 4 .
$$

This case is similar to Case II-(3). The same translation induces an automorphism $f_{r^{\prime}}$ whose action on $\left\{G_{i j}, E_{i}, F_{j}, D_{k}\right\}$ coincides with that of Case II-(3). Since $r^{\prime}$ depends only on $\left\{G_{i j}, E_{i}, F_{j}, D_{k}\right\}, f_{r^{\prime}}$ sends $r^{\prime}$ to $-r^{\prime}$.

Case III-(3). We assume that

$$
r^{\prime}=\left(2 \sum\left(E_{i}+F_{i}+D_{i}+C_{i}\right)+\sum G_{i j}-3\left(E_{1}+E_{2}+D_{2}+D_{4}+C_{1}+C_{2}\right)\right) / 6 .
$$

This case is similar to Case II-(4). We use the same notation as in Case II-(4). Consider the elliptic pencil $\pi$ defined by the same linear system as in Case II-(4). 
$\pi$ has a singular fiber of type $I_{16}$, and $C_{3}, C_{4}$ are contained in other singular fibers. We shall see that the other fixed curve $C$ is smooth too. It suffices to see that both $C_{3}, C_{4}$ are not fixed curves (Then by the same argument as in Case II-(4) we can see that $C=F$ is smooth.) Assume that $C_{3}$ is fixed by $f_{r^{\prime}}$. Then $F$ is reducible and of type $I_{4}$. Thus $\pi$ has singular fibers of type $I_{16}$ and of type $I_{4}$, and four sections. By Shioda 14, Corollaries 1.5, 1.7, $\operatorname{det}\left(S_{X}\right)=4$, which is a contradiction. Thus we have the same configuration of fixed curves as in Mukai and Namikawa [10], (4.2). As stated in II-(4), $f_{r^{\prime}}$ acts trivially on the sublattice of rank 18 generated by 20 nodal curves, and acts as -1 on its orthogonal complement. Hence $f_{r^{\prime}}$ sends $r^{\prime}$ to $-r^{\prime}$.

4.4. Case IV-(1). We assume that

$$
r^{\prime}=\left(2 \sum\left(E_{i}+F_{i}+D_{i}\right)+\sum G_{i j}-4 \sum D_{i}\right) / 8 .
$$

This case is similar to Case II-(1). The involution $f_{r^{\prime}}$ induced by the involution $\left(1_{E_{\sqrt{-1}}},-1_{E_{\sqrt{-1}}}\right)$ acts trivially on the sublattice of rank 18 generated by $\left\{G_{i j}, E_{i}, F_{j}\right\}$. It follows from Mukai and Namikawa [10, Proposition 2.5, that

$$
\operatorname{rank}\left(H^{2}(X, \mathbb{Z})^{\left\langle f_{r^{\prime}}\right\rangle}\right)=18 .
$$

Recall that $r^{\prime}$ is orthogonal to $G_{i j}, E_{i}, F_{j}$ (see the proof of Lemma 3.6, Case IV-(1)). Hence $f_{r^{\prime}}$ sends $r^{\prime}$ to $-r^{\prime}$.

Case IV-(2). We assume that

$$
\begin{aligned}
r^{\prime}= & \left(2 \sum\left(E_{i}+F_{i}+D_{i}\right)+4 \sum L_{j}+\sum G_{i j}\right. \\
& \left.-8\left(L_{1}+L_{4}+L_{6}+L_{7}+L_{10}+L_{11}\right)\right) / 16 .
\end{aligned}
$$

This case is similar to Case II-(2). The same elliptic pencil $\pi$ has three singular fibers of type $I_{6}$, one singular fiber of type $I_{2}$ and eighteen sections. The same translation induces an automorphism $f_{r^{\prime}}$ whose action on $\left\{L_{i}\right\}$ is as follows (the action of $f_{r^{\prime}}$ on $\left\{G_{i j}, E_{i}, F_{j}, D_{k}\right\}$ is the same as in Case II-(2)):

$$
\begin{aligned}
f_{r^{\prime}}\left(L_{5}\right)= & L_{9}, \quad f_{r^{\prime}}\left(L_{9}\right)=L_{1}, \quad f_{r^{\prime}}\left(L_{2}\right)=L_{8}, \\
f_{r^{\prime}}\left(L_{8}\right)= & L_{4}, \quad f_{r^{\prime}}\left(L_{3}\right)=L_{12}, \quad f_{r^{\prime}}\left(L_{12}\right)=L_{6}, \\
f_{r^{\prime}}\left(L_{1}\right)= & G_{11}-L_{5}+\left(G_{12}+G_{21}+E_{3}+F_{4}+D_{3}+D_{4}-L_{2}-L_{3}\right) / 2 \\
& +L_{8}+L_{9}+L_{12}, \\
f_{r^{\prime}}\left(L_{4}\right)= & G_{11}-L_{2}+\left(G_{12}+G_{22}+E_{3}+E_{4}+F_{3}+D_{4}-L_{3}-L_{5}\right) / 2 \\
& +L_{8}+L_{9}+L_{12}, \\
f_{r^{\prime}}\left(L_{6}\right)= & G_{11}-L_{3}+\left(G_{21}+G_{22}+E_{4}+F_{3}+F_{4}+D_{3}-L_{2}-L_{5}\right) / 2 \\
& +L_{8}+L_{9}+L_{12} .
\end{aligned}
$$

Note that

$$
\left|G_{12}+G_{21}+G_{22}+L_{7}+L_{10}+L_{11}\right|=\left|G_{24}+G_{32}+G_{43}+L_{2}+L_{3}+L_{5}\right| .
$$

A direct calculation shows that $f_{r^{\prime}}\left(r^{\prime}\right)=-s^{\prime}$, where

$$
\begin{aligned}
s^{\prime}= & \left(2 \sum\left(E_{i}+F_{i}+D_{i}\right)+4 \sum L_{j}+\sum G_{i j}\right. \\
& \left.-8\left(L_{2}+L_{3}+L_{5}+L_{7}+L_{10}+L_{11}\right)\right) / 16 .
\end{aligned}
$$


Case IV-(3). We assume that

$$
\begin{aligned}
r^{\prime}= & \left(2 \sum G_{i j}+\sum\left(E_{i}+F_{i}+D_{i}\right)-\sum L_{j}\right. \\
& -2\left(G_{11}+G_{13}+G_{22}+G_{24}+G_{31}+G_{33}+G_{42}+G_{44}\right) \\
& \left.+4\left(L_{1}+L_{2}+L_{5}+L_{7}\right)\right) / 8
\end{aligned}
$$

This case is similar to Cases II-(4) and III-(3). By the same argument, we have an involution $f_{r^{\prime}}$ which sends $r^{\prime}$ to $-r^{\prime}$.

Case IV-(4). We assume that

$$
\begin{aligned}
r^{\prime}= & \left(5 \sum G_{i j}+20 \sum F_{i}+10 \sum L_{i}-16\left(G_{22}+G_{24}+G_{41}+G_{43}\right)\right. \\
& \left.-24\left(F_{2}+F_{4}+L_{2}+L_{5}+L_{8}+L_{11}\right)\right) / 16
\end{aligned}
$$

In this case there are exactly twenty-two nodal curves orthogonal to $r^{\prime}$ whose dual graph is the same graph as that of Case III-(3). Thus we have an involution $f_{r^{\prime}}$ which sends $r^{\prime}$ to $-r^{\prime}$.

\section{The AUtomorphism Groups}

In this section we shall complete a calculation of automorphism groups. First recall that the graph of Leech roots as in Lemma 3.2 is isomorphic to the dual graph $\Gamma$ of nodal curves on $X$ (see $\S 2)$. Since $w$ is an interior point of $D, w^{\prime}$ is also an interior point of a fundamental domain of $W\left(S_{X}\right)^{(2)}$. Hence, modulo $W\left(S_{X}\right)^{(2)}$, we may assume that $w^{\prime}$ is contained in the Kähler cone $D(X)$, i.e. it is represented by a class of an ample divisor of $X$. Then each Leech root $r$ as above is represented by an irreducible curve, because $\left\langle r, w^{\prime}\right\rangle=1$. Hence these Leech roots are represented by nodal curves. Since $A u t(X)$ depends only on the isomorphism class of the lattice $S_{X}$ (see Lemma 1.1), we can identify these Leech roots with nodal curves as in $\S 2$.

Next we remark that the subgroup $G_{0}$ of $A u t\left(D^{\prime}\right)$ (see Lemma 3.5) can be represented by automorphisms of $X$. Since $G_{0}$ acts on the discriminant $A_{S_{X}}$ trivially, the action of $G_{0}$ on $S_{X}$ can be extended to the one on $H^{2}(X, \mathbb{Z})$ acting on $T_{X}$ trivially. Hence the assertion follows from the Torelli theorem for $K 3$ surfaces (Piatetski-Shapiro and Shafarevich [13]).

Case I. We first remark that $O\left(q_{S_{X}}\right)$ is isomorphic to $O^{+}\left(4, \mathbb{F}_{2}\right) \simeq\left(S_{3} \times S_{3}\right) \cdot \mathbb{Z} / 2 \mathbb{Z}$. Let $\iota$ be an automorphism of $X$ induced from an automorphism of $E \times F$ given by

$$
(x, y) \longrightarrow(x,-y) .
$$

It is easy to see that the pointwise fixed curves of $\iota$ are exactly $E_{i}, F_{j}$. It is known (Mukai and Namikawa [10]) that $\iota^{*} \mid S_{X}=1$ and $\iota^{*} \mid T_{X}=-1$. This implies that $\iota$ is contained in the center of $A u t(X)$. The quotient surface $X /\langle\iota\rangle$ contains sixteen exceptional curves which are the images of $\left\{G_{i j}\right\}$. By contracting these exceptional curves, we have a smooth quadric $Q$. Thus $A u t(X) /\langle\iota\rangle$ is isomorphic to a subgroup of the group of birational transformations of $Q$. Denote by $\left\{t_{i j}\right\}$ involutions of $X$ induced from the translations of $E \times F$ by 2-torsions $\left(p_{i}, q_{j}\right)$. Then $\iota$ and $t_{i j}$ generate a 2-elementary abelian group of order 32. By Lemma 3.5, we have

Lemma 5.1. Aut $\left(D^{\prime}\right)$ is generated by sixteen involutions $t_{i j}$ and $O^{+}\left(4, \mathbb{F}_{2}\right)$.

Let $N_{1}$ be a subgroup of $\operatorname{Aut}(X)$ generated by twenty-four involutions $f_{r^{\prime}}$ corresponding to the twenty-four Leech roots in Lemma 3.6 (see 4.1 for $f_{r^{\prime}}$ ). 
Lemma 5.2. Let $\phi$ be an isometry of $S_{X}$ which preserves the Kähler cone $D(X)$. Then there exists an element $g \in N_{1}$ such that $g \circ \phi \in A u t\left(D^{\prime}\right)$.

Proof. Since $f_{r^{\prime}}\left(r^{\prime}\right)=-r^{\prime}$ (see 4.1), $f_{r^{\prime}}$ sends the positive side with respect to the hyperplane defined by $r^{\prime}$ to the opposite side. Thus the same proof as in Kondo $[8$, Lemma 5.3, holds. We remark that this proof works in the remaining Cases II-IV by using the fact that $f_{r^{\prime}}\left(r^{\prime}\right)=-r^{\prime}$ or $f_{r^{\prime}}\left(r^{\prime}\right)=-s^{\prime}$, where $s^{\prime}$ is the projection of a Leech root $s$ of the same type (see $\S 4$, Case II-(2), Case III-(1), Case IV-(2)).

Theorem 5.3. The automorphism group of $K m(E \times F)$ is a split extension of the 2 -elementary abelian group $(\mathbb{Z} / 2 \mathbb{Z})^{5}$ of order 32 by a group $N_{1}$ where $(\mathbb{Z} / 2 \mathbb{Z})^{5}$ is generated by translations $t_{i j}$ and $\iota$, and $N_{1}$ is generated by twenty-four involutions $f_{r^{\prime}}$ from Cremona transformation of $\mathbb{P}^{2}$.

Proof. By the definition of genericness of $X, \operatorname{Aut}(X) \cap \operatorname{Aut}\left(D^{\prime}\right)$ is a 2-elementary group of order 32 generated by $t_{i j}$ and $\iota$ (see Lemmas 1.1, 5.1). On the other hand, for each $f \in \operatorname{Aut}(X)$, there exists a $g \in N_{1}$ with $g \circ f \in \operatorname{Aut}(X) \cap \operatorname{Aut}\left(D^{\prime}\right)$ (Lemma $5.2)$. Thus we have proved the assersion.

Case II. In this case, $G_{0}$ is generated by $\left\{t_{i j}\right\}$ and an automorphism $\tau$ of order three (see the proof of Lemma 3.5, Case II). Let $\sigma$ be an involution of $X$ induced from

$$
(x, y) \longrightarrow(y, x)
$$

which acts on $T_{X}$ as $-1_{T_{X}}$. Let $N_{2}$ be a subgroup of $A u t(X)$ generated by $f_{r^{\prime}}$ corresponding to the Leech roots as in Lemma 3.6 (also see 4.2 for $f_{r^{\prime}}$ ). Then, by the same argument as in Case I, we have

Theorem 5.4. The automorphism group of $K m(E \times E)$ is a split extension of $\left((\mathbb{Z} / 2 \mathbb{Z})^{4} \cdot \mathbb{Z} / 3 \mathbb{Z}\right) \cdot \mathbb{Z} / 2 \mathbb{Z}$ by $N_{2}$, where the former group is generated by $t_{i j}, \sigma$ and $\tau$.

Remark 5.5. $\iota$ defined in Case I does not preserve $D^{\prime}$ and is contained in $N_{2}$ (see 4.2, Case II-(1)).

Case III. In this case, $O\left(q_{S_{X}}\right) \simeq S_{3} \times \mathbb{Z} / 2 \mathbb{Z}$ and $G_{0}$ is generated by $\left\{t_{i j}\right\}$ and automorphisms induced from

$$
(x, y) \longrightarrow(y,-x+y),(x, y) \longrightarrow\left(\omega(-x+y),-\omega^{2} y\right) .
$$

We shall find an automorphism of order 6 which preserves $D^{\prime}$ and whose image in $O\left(q_{S_{X}}\right)$ has order 6 (see Lemma 1.1). Let $\sigma$ be an involution of $S_{X}$ defined by

$$
\begin{aligned}
& E_{1} \leftrightarrow G_{24}, \quad E_{2} \leftrightarrow G_{43}, \quad E_{3} \leftrightarrow G_{32}, \quad E_{4} \leftrightarrow G_{11}, \\
& F_{1} \leftrightarrow G_{44}, \quad F_{2} \leftrightarrow G_{31}, \quad F_{3} \leftrightarrow G_{23}, \quad F_{4} \leftrightarrow G_{12}, \\
& D_{1} \leftrightarrow G_{14}, \quad D_{2} \leftrightarrow G_{42}, \quad D_{3} \leftrightarrow G_{21}, \quad D_{4} \leftrightarrow G_{33}, \\
& C_{1} \leftrightarrow G_{34}, \quad C_{2} \leftrightarrow G_{22}, \quad C_{3} \leftrightarrow G_{41}, \quad C_{4} \leftrightarrow G_{13} .
\end{aligned}
$$

Note that $\sigma$ interchanges two families $\left\{G_{i j}\right\}$ and $\left\{E_{i}, F_{j}, D_{k}, C_{l}\right\}$, and $\sigma$ is in fact an isometry of $S_{X}$ because these curves generate $S_{X}$ (Lemma 2.1). Recall that 
$A_{S_{X}}=S_{X}^{*} / S_{X} \simeq(\mathbb{Z} / 2 \mathbb{Z})^{4} \times \mathbb{Z} / 3 \mathbb{Z}$. Since $\sigma$ is an involution, it acts on the 2-Sylow subgroup of $A_{S_{X}}$ trivially. On the other hand we can see that the class

$$
\delta=\sum\left(G_{i j}+2\left(E_{k}+F_{l}+D_{m}+C_{n}\right)\right) / 3
$$

is a generator of the 3-Sylow subgroup of $A_{S_{X}}$, and $\sigma(\delta)=-\delta$. Thus $\sigma$ can be extended to an isometry of $H^{2}(X, \mathbb{Z})$ with $\sigma \mid T_{X}=-1_{T_{X}}$. Since $\sigma$ preserves the projection of the Weyl vector (Lemma 3.4), it preserves the Kähler cone too. It now follows from the Torelli theorem that $\sigma$ is represented by an automorphism of order two. Let $\tau$ be an automorphism of order 3 induced from

$$
(x, y) \longrightarrow(\omega x, \omega y) .
$$

Then $\sigma \circ \tau$ is the desired automorphism.

Let $N_{3}$ be a subgroup of $\operatorname{Aut}(X)$ generated $f_{r^{\prime}}$ corresponding to the Leech roots as in Lemma 3.6. Then we have

Theorem 5.6. The group of automorphisms of $K m\left(E_{\omega} \times E_{\omega}\right)$ is a split extension of $\left((\mathbb{Z} / 2 \mathbb{Z})^{4} \cdot A_{4}\right) \cdot \mathbb{Z} / 6 \mathbb{Z}$ by $N_{3}$.

Case IV. In this case, $O\left(q_{S_{X}}\right) \simeq D_{8}$ and $G_{0}$ is generated by translations by sections of five elliptic pencils with six singular fibers of type $I_{4}$. On the other hand, as we mentioned in the proof of Lemma 3.5, there is an isometry in $\operatorname{Aut}(D)$ acting on $R=A_{3} \oplus A_{3}$ as automorphism of order 4. This induces an isometry $\phi$ of $S_{X}$ preserveing $D^{\prime}$. Then $\phi$ can be extended an isometry of $H^{2}(X, \mathbb{Z})$ acting on $T_{X}$ as an isometry of order 4 . It now follows from the Torelli theorem that $\phi$ is represented by an automorphism of order 4 .

Let $N_{4}$ be a subgroup of $A u t(X)$ generated by $f_{r^{\prime}}$ corresponding to the Leech roots as in Lemma 3.6. Then we have

Theorem 5.7. The group of automorphisms of $K m\left(E_{\sqrt{-1}} \times E_{\sqrt{-1}}\right)$ is a split extension of $\left((\mathbb{Z} / 2 \mathbb{Z})^{4} \cdot A_{5}\right) \cdot \mathbb{Z} / 4 \mathbb{Z}$ by $N_{4}$.

Remark 5.8. Let $M_{24}$ be the Mathieu group, which acts naturally on a set $\Omega$ of twenty-four letters. Let $M_{20}$ be the pointwise stabilizer group of four points in $\Omega$. Then $M_{20}$ is isomorphic to $(\mathbb{Z} / 2 \mathbb{Z})^{4} \cdot A_{5}$. In $[9]$ the second author has proved that $\left((\mathbb{Z} / 2 \mathbb{Z})^{4} \cdot A_{5}\right) \cdot \mathbb{Z} / 4 \mathbb{Z}$ has the maximum order among all finite groups of automorphisms of $K 3$ surfaces.

Remark 5.9. The method in this paper is applicable to other $K 3$ surfaces, for example, the Fermat quartic surface. In this case there exists a primitive embedding of the Picard lattice into $I I_{1,25}$ such that forty-eight lines on the Fermat quartic surface appear as a part of faces of the corresponding $D^{\prime}$. The remaining problem is to find an automorphism corresponding to each of the remaining faces of $D^{\prime}$ like $f_{r^{\prime}}$ as above.

\section{ACKNOWLEDGEMENTS}

A part of this paper was written during the first author's stay at University of Michigan and the second author's stay at the Max-Planck-Institut für Mathematik in Bonn. They express their thanks to both Institutions. 


\section{REFERENCES}

[1] R. Borcherds, Automorphism groups of Lorentzian lattices, J. Algebra 111 (1987), 133153. MR 89b:20018

[2] J. H. Conway, Three lectures on exceptional groups, in Finite simple groups, Academic Press, 1971, pp. 215-247. MR 49:2918

[3] J. H. Conway, The automorphism group of the 26 dimensional even Lorentzian lattice, J. Algebra 80 (1983), 159-163. MR 85k:11030

[4] J. H. Conway, N.J.A. Sloane, Sphere packings, lattices and groups, Grundlehren Math. Wiss. Bd 290, 2nd ed. 80 (1988). MR 89a:11067

[5] J. H. Keum, Automorphisms of Jacobian Kummer surfaces, Compositio Math. 107 (1997), 269-288. MR 98e:14039

[6] J. H. Keum, Every algebraic Kummer surface has infinitely many automorphisms, unpublished manuscript (1996).

[7] S. Kondō, Enriques surfaces with finite automorphism groups, Japanese J. Math. 12 (1986), 191-282. MR 89c:14058

[8] S. Kondō, The automorphism group of a generic Jacobian Kummer surface, J. Algebraic Geometry 7 (1998), 589-609. MR 99i:14043

[9] S. Kondō, The maximum order of finite groups of automorphisms of K3 surfaces, Amer. J. Mathematics 121 (1999), 1245-1252.

[10] S. Mukai, Y. Namikawa, Automorphisms of Enriques surfaces which act trivially on cohomology groups, Invent. math. 77 (1984), 383-397. MR 86i:14012

[11] V.V. Nikulin, Finite groups of automorphisms of Kählerian surfaces of type K3, Moscow Math. Soc. 38 (1980), 71-137. MR 81e:32033

[12] V.V. Nikulin, Integral symmetric bilinear forms and their applications, Math. USSR Izv. 14 (1980), 103-167. MR 80j:10031

[13] I. Piatetski-Shapiro, I.R. Shafarevich, A Torelli theorem for algebraic surfaces of type K3, Math. USSR Izv. 5 (1971), 547-587. MR 44:1666

[14] T. Shioda, On elliptic modular surfaces, J. Math. Soc. Japan 24 (1972), 20-59. MR 55:2927

[15] T. Shioda, H. Inose, On singular K3 surfaces, Complex analysis and algebraic geometry (Collection Dedicated to K. Kodaira), Iwanami Shoten, Tokyo, and Cambridge Univ. Press, Cambridge (1977), 119-136. MR 56:371

[16] J.A. Todd, A representation of the Mathieu group $M_{24}$ as a collineation group, Ann. Mat. Pure Appl. 71 (1966), 199-238. MR 34:2713

[17] E.B. Vinberg, Some arithmetic discrete groups in Lobachevskii spaces, in Proc. Int. Coll. on Discrete Subgroups of Lie Groups and appl. to Moduli (Bombay 1973), Oxford Univ. Press, 1975., pp. 323-348. MR 54:10492

Department of Mathematics, Konkuk University, Seoul 143-701, Korea and Korea Institute for Advanced Study, Seoul 130-012, Korea

E-mail address: jhkeum@kkucc.konkuk.ac.kr

Current address: Korea Institute for Advanced Study, 207-43 Cheongryangri-dong, Dongdaemun-gu, Seoul 130-012, Korea

E-mail address: jhkeum@kias.re.kr

Graduate School of Mathematics, Nagoya University, Nagoya 464-8602, Japan

E-mail address: kondo@math.nagoya-u.ac.jp 